P96 (continued)

ciated with having an overweight/obese child as communication barriers. HS (eg, consulting dietitian) and community-based (eg, WIC) educational/counseling opportunities for families were identified as supportive of Manager efforts to communicate about children's weight status.

Conclusions: Participating HS programs acknowledged the importance of communicating with parents about their children's weight status. However, findings also indicated parents' personal feelings about their child's weight status may influence the type of communication they receive and their willingness to utilize other educational/counseling opportunities. More research is needed to explore parents' perceptions of HS communication methods, as well as effective strategies for supporting and educating parents about healthy weight in childhood.

Funding: East Carolina University Graduate Student Thesis Award.

\section{P97 Mealtime Emotional Climate and Child Health: A Systematic Review}

Jasmin Smith, MS, jasmith@huskers.unl.edu, University of Nebraska-Lincoln, 205 Louise Pound Hall, 512 N 12 St, Lincoln, NE, 68588-0366; Dipti Dev, PhD, University of Nebraska-Lincoln; Jaclyn Saltzman, PhD, MPH, MITRE Health FFRDC

Background: Positive mealtime emotional climate (MEC) has been linked to better nutrition, psychosocial, literacy and academic outcomes, and fewer behavior problems. However, MEC has been defined in a variety of ways across studies, limiting the ability to synthesize findings and plan future research.

Objective: To determine how previous studies have measured MEC, and to identify which child characteristics are associated with MEC.

Study Design, Setting, Participants: We searched 3 databases (1980-2018) for peer-reviewed articles measuring MEC. Inclusion criteria required at least 1 child-level outcome related to physical, nutritional, or developmental health; children aged 0-18 years old; and quantitative data using cohort, case-control, intervention, or experimental designs.

Measurable Outcome/Analysis: To address the first objective, we extracted data about the measures and definitions of MEC from each included article. To address the second aim, we used a previously published taxonomy to categorize child/adolescent characteristics according to the amount of evidence linking them to MEC. A characteristic was categorized as a "correlate" (positive or negative association) or "non correlate" (no association) if $>3$ studies assessing that characteristic found consistent results. If $>3$ studies assessing a characteristic had inconsistent results, the construct was classified as "unclear." If $<2$ studies assessed a characteristic, then it was not categorized due to an inadequate amount of evidence.
Results: Out of 561 unique studies identified in the initial search, 13 met inclusion criteria, and only 3 used the same measure of MEC. Healthful dietary intake, disordered eating behaviors, and weight/BMI were categorized as correlates of MEC, but links to unhealthy dietary intake are unclear. Several characteristics (eg, temperament, academic success) were examined in only 1 study.

Conclusions: More research is needed to understand the relationship between MEC and child developmental outcomes and physical activity. These findings aid in conceptualizing how MEC has been defined and measured and illuminate the importance of MEC on children's health.

Funding: USDA.

\section{P98 Student Perceptions of Food and Climate Change}

Deanne Moosman, MS, moosmandl@vmi.edu, Virginia Military Institute, 223 Cormack Hall, Lexington, VA, 24450

Background: Many recent reports recommend shifting from a meat-centric diet to a plant-based diet to support sustainability and carbon emission goals. More research is needed to assess whether college students' concerns regarding climate change influence their food selection and whether they have the knowledge and willingness to make dietary changes to reduce their carbon footprint.

Objective: To determine the attitudes and perceptions of food and climate change of college freshmen attending a military college and to examine ways they are willing to change their diet.

Study Design, Setting, Participants: College freshmen (99 males, 19 females; ages 18-21) attending a military school were selected from a required health class to participate in a 60-item questionnaire, before any topics regarding nutrition were taught. Data collected included eating habits, willingness to adopt various diets, concerns regarding food availability, and knowledge/perceptions of climate change.

Measurable Outcome/Analysis: Data were analyzed using descriptive statistics.

Results: Many students surveyed (83\%) feel they have an average or better understanding of climate change; however, only $44 \%$ agreed that the selection/consumption of certain foods contribute to climate change. Over half the students surveyed were concerned how climate change will impact them. A majority of those surveyed consume beef either daily (36\%) or weekly $(57 \%)$. The primary reason for eating beef was to meet protein requirements and most respondents (73\%) felt adopting a plant-based diet would not sufficiently meet those needs. Despite cattle having a large carbon footprint, respondents want to be able to consume beef (42\%) and dairy (48\%) whenever they want, without restrictions. Survey respondents are more willing to replace beef with poultry, fish, or game rather than adopt a vegan, vegetarian, or partially plantbased diet. 
P98 (continued)

Conclusions: This population of students may benefit from educational outreach that provides them with a better understanding of how food selection can impact climate change, and how protein requirements can be met even when meat is partially or fully replaced with various plant sources.

Funding: None.

\section{Behavior and Education Theory}

\section{P99 Improved Self-efficacy and Nutrition Knowledge Among Indiana High School Teachers After Training in and Implementation of a New Nutrition Curriculum}

Elizabeth Kaschalk, MA, Indiana University; Elizabeth Foland, MS, RD, Indiana Department of Education-Office of School and Community Nutrition; Alyce Fly, PhD, afly@indiana.edu, Indiana University, 1025 E 7th St \#116, Bloomington, IN, 47405

Background: Professional development can improve teacher self-efficacy and knowledge to enhance their delivery of food guidance. This may result in improvements in students' knowledge about and skills for healthy eating.

Objective: To explore effects of a training on a nutrition curriculum, Forecasting Your Future: Nutrition Matters, on teachers' self-efficacy to teach nutrition and knowledge of 2015 Dietary Guidelines. To explore teachers' outcome expectations for students.

Study Design, Setting, Participants: Family and Consumer Sciences high school teachers were recruited for training at a state conference and divided into 9-strata based on school location and size, then randomly assigned to intervention (INT, $\mathrm{n}=18$ ) or control (CON, $\mathrm{n}=18)$. INT were trained (spring 2018), then implemented the new curriculum in the classroom (fall 2018); CON implemented the usual curriculum. Both completed a 60-item pre-test (spring 2018) and 55-item post-test (late fall 2018). Follow-up semi-structured telephone interviews were conducted $(n=32)$ to expand on multi-opt results. Teachers answered 6 open-ended questions about outcome expectations for their students.

Measurable Outcome/Analysis: Survey data were analyzed by hierarchical linear modeling to determine whether changes from INT differed from CON for self-efficacy to teach nutrition and nutrition knowledge. Interviews were recorded, transcribed and imported into NVivo software, then thematically analyzed.

Results: After training then teaching the curriculum, INT had greater increases in nutrition knowledge of the 2015 Dietary Guidelines $(P=.028)$ and self-efficacy to teach nutrition $(P=.010)$ vs CON. Interviews with INT revealed they did not expect immediate behavior changes but hoped to affect students' future health by giving them the knowledge and skills necessary to make healthy choices.

Conclusions: Professional development sessions and providing updated curricula has promise for improving teachers' nutrition knowledge and self-efficacy to teach nutrition. More research should be done on outcome expectations to guide future curriculum development.

Funding: USDA Team Nutrition.

\section{Basic Food and Nutrition Knowledge}

\section{P100 Exploring the Concept of Sustainability in Nutrition and Dietetics: Student, Academic and Practitioner Perspectives}

Sarah Burkhart,PhD, sburkhar@usc.edu.au, University of the Sunshine Coast, Locked Bag 4, Maroochydore, Queensland, Australia, 4556; Michele Verdonck, PhD, University of the Sunshine Coast; Theresa Ashford, PhD, University of the Sunshine Coast; Judith Maher, PhD, University of the Sunshine Coast

Background: Nutrition and dietetic (N\&D) professionals can contribute to sustainable development and achievement of the United Nations' Sustainable Development Goals. However, there are diverse understandings of what sustainability means in the N\&D context which may be constraining higher education curricula.

Objective: To explore the concept of 'sustainability' in the context of N\&D from student, academic, and practitioner perspectives.

Study Design, Setting, Participants: An action research process was used to explore the concept of sustainability. Data was collected sequentially, first from undergraduate students' self-reported perceptions of sustainability ( $n=95$, self-administered online survey, open question) and then academics' perceptions of sustainability-related teaching practice $(n=7$, workshop) at a regional Australian university. Semi-structured interviews were then undertaken with Australian N\&D practitioners $(n=10)$ having longevity in the dietetic/nutrition profession as well as a specialist practice area. Data was analysed concomitantly and used as a sensitising device with each subsequent group.

Measurable Outcome/Analysis: Students provided 4 keywords/phrases conveying their personal understanding of sustainability within the N\&D context. Academics provided a written response to the question; how do you perceive sustainability? Practitioners answered questions from an interview protocol developed to enable exploration of sustainability both conceptually and in practice. Each data set was analysed for theme by 2 independent researchers. Findings across participant groups were then analysed for overarching themes.

Results: Across groups, sustainability was perceived as a broad, future-focused concept, multi-factorial (environment, culture, social, economic), and relating to dietary recommendations and practices. Themes from student responses were sustainable food systems and system influencers. Academic and practitioner themes diverged to include sustaining the profession, sustaining the effects of our work, and stewardship of resources. Sustainability was 